\title{
Fasting Insulin Level Changes after Large Volume Liposuction
}

\author{
Hanna Habib, Sarah Abd Alazeem, Nermin Abd Alazeem
}

\author{
Department of General Surgery, Faculty of Medicine - Ain Shams University
}

Corresponding author: Nermin A. Abdelaziz; Mobile: 01013613439; Email: nerminabdelaziem@gmail.com

\begin{abstract}
Background: Obesity is a disabling disease which has gained greater attention worldwide. It significantly increases the risk for other diseases such as insulin independent diabetes mellitus also known as diabetes type 2 . The most common surgical procedure for obesity is liposuction. It is traditionally performed either as small-volume liposuction or large-volume liposuction. Aim of the Work: to spot the light on the effect of large volume liposuction on fasting insulin level changes after 3 months postoperative and to find if LVL may obtain any improvement in metabolic variables. This study will provide the clinician with a more valid basis on which to advise patients undergoing bodycontouring procedures, particularly to ensure realistic expectations regarding the effects of bodyreshaping procedures on general health. Patients and Mehtods: This is prospective study was conducted on a total of 15 overweight and obese (BMI $26-35 \mathrm{~kg} / \mathrm{m}^{2}$ ) premenopausal women (age 2140years). All subjects were at their maximum body weight and weight stable for at least 3 months. The study took place at Ain Shams University Hospitals and other authorized hospitals under supervision of thesis supervisors studying fasting insulin changes after 3 months following large volume liposuction. Results: The present study demonstrates that large-volume abdominal liposuction should, by itself, be considered a clinical therapy for obesity and its metabolic sequelae. Aspiration of large amounts of subcutaneous abdominal fat in women with abdominal obesity, besides having cosmetic benefits, does significantly improve fasting insulin levels. Therefore, the procedure is safe and could successfully help obese subjects to reduce their potential metabolic risks. Conclusion: The analysis of the study suggests that plastic surgery could play a role in metabolism. The surgical removal of fat is not detrimental with regard to variation of metabolic indices.
\end{abstract}

Key words: Fasting insulin, large volume liposuction, body contouring, obesity.

\section{Introduction}

Obesity is increasingly frequent in our society and is associated closely with cardiovascular risk factors and metabolic disorders ${ }^{(\mathbf{1})}$.

Liposuction has become one of the most popular cosmetic procedures performed. The vast number of potential candidates, the relative ease of performance, the safety, and the high patient satisfaction rate with suction lipectomy are the primary reasons for its popularity.The goal of the liposuction surgeon is to remove "target" fat, leaving the desired body contour and smooth transitions between suctioned and nonsuctioned areas ${ }^{(2)}$.

Given that the procedure is traditionally performed for cosmetic reasons, there are no specific recommendations with respect to appropriate degree of obesity for the procedure, and suction lipectomy is often performed on individuals with a BMI in the normal or overweight categories ${ }^{(3)}$.

Liposuction is done by several techniques including introduction of tumescent, ultrasonic liposuction, power-assisted liposuction, and, more recently, laser-assisted liposuction) ${ }^{(4)}$.

Liposuction classified into two types according to the volume of solution aspirated: High volume or low volume $(<4,000 \mathrm{ml}$ aspirated) ${ }^{(5)}$.

The definition of "large volume liposuction" varies in the plastic surgery literature. In fact, no strict definition exists. The most common definitions refer to either total fat removed during the procedure $(\mathrm{eg}, 4 \mathrm{~L}$ of fat removal) or total volume removed during the procedure (fat plus wetting solution, eg, $5 \mathrm{~L}$ of total volume removal (fat plus wetting solution) (๑).

Recently, it has been suggested large volume liposuction is a potential treatment for reducing metabolic complications due to obesity (7).

Insulin is an anabolic hormone that regulates plasma glucose by promoting glucose uptake, glycogenesis, lipogenesis, and protein synthesis of skeletal muscle and fat 
tissue through the tyrosine kinase receptor pathway. Fasting insulin levels provide the most utility as a clinical tool because the highest ratios suggest insulin resistance and significantly greater likelihood of identifying risk for type 2 diabete ${ }^{(8)}$.

Fasting insulin level can be determined by a simple blood test after fasting 8 hours. A normal fasting blood insulin level is below $8.4 \mathrm{microU} / \mathrm{m}$, but some studies consider it safe below $5 \mathrm{microU} / \mathrm{ml}^{(9)}$.

Insulin sensitivity which describes how sensitive the body is to the effects of insulin has been shown to be negatively correlated with the amount of deep subcutaneous adipose tissue. The deeper subcutaneous adipose tissue has also been shown to be metabolically active, Up to $50 \%$ of the subcutaneous adipose tissue is in the deep compartment, and this tissue is removed with liposuction ${ }^{(23)}$. That is why if we do liposuction to deep adipose layer we suspect positively influenced fasting plasma insulin and insulin sensitivity, subsequently liability to have future risk for type 2 diabetes is decreased ${ }^{(\mathbf{1 0})}$.

\section{Aim of the Work}

This a prospective study aiming study to spot the light on the effect of large volume liposuction on fasting insulin level changes after 3 months postoperative And to find if LVL may obtain any improvement in metabolic variables. This study will provide the clinician with a more valid basis on which to advise patients undergoing body-contouring procedures, particularly to ensure realistic expectations regarding the effects of body-reshaping procedures on general health.

\section{Patients and Methods}

\section{Study design:}

This is a prospective study which was conducted at Ain Shams University hospitals and other authorized hospitals under supervision of thesis supervisors studying fasting insulin changes after 3 months following large volume liposuction.

A total of 15 overweight and obese (BMI 26-35 $\mathrm{kg} / \mathrm{m}^{2}$ ) premenopausal women (age 21-40years) were enrolled. All subjects were at their maximum body weight and weight stable for at least 3 months

Informed consent was taken from all patients who accepted to participate in the study. Confidentiality is assured of the personal data and medical information of all patients.

Patients:

Inclusion Criteria: Patients ages from 20 to 45 years old with localized fat deposits with.

a. American Society Of

Anaesthesiologist Grading

1. Normal healthy patient(ASA) class I

2. Patients with mild systemic disease, or ASA class II

b. Realistic expectations regarding the outcome of the procedure.

All the subjects had a stable weight (with fluctuations of not more than 2 percent of the body weight) for at least two months and had been sedentary (exercising for less than one hour per week) for at least six months before entering the study.

\section{Exclusion Criteria:}

1. Patients undergoing concomitant procedures.

- Presence of significant medical diseases such as diabetes mellitus, cardiac, renal, hepatic, gastrointestinal or endocrinal diseases (American Society of Anaesthesiologist Grading III and IV) (Patients with severe systemic disease and Patients with severe systemic disease that is a constant threat to life.

Methods: following:

All patients were subjected to the

\section{Preoperative:}

a. Clinical history:

- Personal history: including age, sex, weight, occupation, special habits of medical importance particularly smoking, parity, contraception and menstrual history.

- History of present illness: exclude pregnancy, poor wound healing, Allergic reactions to medications

b. Past history of medical diseases: such as diabetes, infections, malignancy, liver or renal dysfunction, heart disease, history of DVT, past abdominal surgeries.

c. Psychological assessment must be undertaken. Inquires are made about diet and exercise habits and any history of weight gain and loss as this can affect the long term success of the procedure. 
Patients are counselled on the limitations and risks associated liposuction. Their expectations are determined to ensure they are realistic and are aware that full results may take up to 12 weeks to be seen. Liposuction does not result in any significant weight loss and patients should also be aware that fat removed may return if excess weight is put on.

d. Clinical examination:

- General: temperature, heart rate and signs of anemia.

- Local examination:

Inspection: to detect areas involved, symmetrical or ventral hernias.

- Palpation: An assessment of general physical health is necessary to determine whether a patient is a suitable candidate for surgery. The specific sites that are being considered for liposuction are examined for potential problems. Skin tone and elasticity is assessed as well as the presence of hernias, scarring, cellulite and stretch marks. If patients have poor skin elasticity they are informed that following surgery they may have skin draping which may need further surgical correction

- Investigations: Usually a complete blood cell count with quantitative platelet assessment, prothrombin time, partial thromboplastin time, liver function tests, pregnancy test for women of child bearing age are performed, hemoglobin, fasting insulin level, random glucose level, HbAlc.

- Operative technique: Precise and accurate pre operative marking is essential for a good result. With the patient standing, areas to be treated are outlined with a fiber tip permanent marker pen and preoperatively photographed.,

Each subject underwent large-volume tumescent liposuction, defined as the removal of more than 4 liters of aspirate

All patients underwent liposucton from abdomen, plus smaller amounts of fat were removed from the arms, flanks, back, hips, or thighs.

The patient's skin is painted with $5 / 10$ percent Povidone Iodine solution while he/she stands next to a sterile draped operating table.

\section{Tumescent infiltration}

- All areas to be treated are injected with Tumescent formula used consists of:

- 1 liter of ringer lactate.
- $1 \mathrm{ml}$ adrenaline (1:1000).

- lidocaine (final dose $50.471 .4 \mathrm{mg} / \mathrm{kg}$ )

Till turgour of the tissues is appreciable equally on both sides. Effective vasoconstriction is achieved in about ten to fifteen minutes, but the effect is more pronounced after about twenty minutes

Tumescent fluid (maximum of $12,500 \mathrm{cc}$ in the series)

\section{Aspiration}

- Access incisions of size $1.5 \mathrm{~cm}$ are made at the periphery of operative field in concealed areas.

- Aspiration begins 20 minutes after infiltration. Deeper areas and areas with more voluminous fat deposits are aspirated using cannulae of 5 or $6 \mathrm{~mm}$ diameter. Smaller fat deposits and the more superficial areas are aspirated with cannulae 3 to $4 \mathrm{~mm}$ in diameter.

- The cannulae move parallel to the fat plane with the openings directed away from skin surface in a to and fro motion along the same path. The site is changed when the aspirate tends to become blood stained.

- Feathering of the peripheral areas is done once the basic earmarked areas have been symmetrically contoured bilaterally. The closure of these access incision sites is accomplished with interrupted loose sutures to permit easy drainage of fluid, reduce oedema and seroma.

- The end point of aspiration is determined by the contents and volume of aspirate as also the appearance and feel of the treated area and bilateral symmetry. Aspirate volumes from bilaterally symmetrical areas should be approximately the same, although the volume of the preoperative injection will influence the volume of the aspirate.

- Participants were bandaged and compression garment placed. Following lipectomy, the subject's fast was broken and they were fed a snack.

Postoperative:

a. Clinical assessment:

Patients were monitored to detect early symptoms subjective to post-operative complications;

- Pain which should be temporary and can be ontrolled by either over-the-counter medication. 
- Numbness which may persists for a few weeks.

- Limited mobility.

- Rates of serious or fatal complications are in the range of $0.02 \%$ to $0.3 \%$ and are predominantly attributed to pulmonary embolus, fat embolus, abdominal perforation, anesthesia.

b. Biochemical assessment: $\mathrm{Hb}$ is necessary just post operative, 12 weeks postoperatively $\mathrm{hb}$ and fasting insulin level, $\mathrm{HbA} 1 \mathrm{c}$ are measured

Subjects were instructed to resume their normal lifestyle after the initial recovery period and to weigh themselves weekly at home, maintaining their usual food intake and physical activity in order to maintain a stable body weight.

No serious complications occurred in any subject and all were able to return to their usual lifestyle within 10 days after liposuction. Re-evaluation of BMI and waist circumference was undertaken 10-12 weeks post-operatively.

\section{Statistical analysis:}

Recorded data were analyzed using the statistical package for social sciences, version 20.0 (SPSS Inc., Chicago, Illinois, USA). Quantitative data were expressed as mean \pm standard deviation (SD). Qualitative data were expressed as frequency and percentage.

\section{The following tests were done:}

- Paired sample t-test of significance was used when comparing between related sample.

- Pearson's correlation coefficient (r) test was used to assess the degree of association between two sets of variables

- The confidence interval was set to $95 \%$ and the margin of error accepted was set to $5 \%$. So, the p-value was considered significant as the following:

- Probability (P-value)

- P-value $<0.05$ was considered significant.

- P-value <0.001 was considered as highly significant.

- P-value >0.05 was considered insignificant.

\section{Results:}

Table (1): Demographic data distribution of the study group.

\begin{tabular}{||l|l||}
\hline Demographic data & Total $(\mathbf{N}=\mathbf{1 5})$ \\
\hline \hline Sex & $15(100 \%)$ \\
$\quad$ Female & $0(0 \%)$ \\
$\quad$ Male & \\
\hline Age (years) & $7(46.7 \%)$ \\
$\quad \leq 30$ years & $8(53.3 \%)$ \\
$\quad>30$ years & $21-40[31.27 \pm 5.69]$ \\
\hline Range [Mean \pm SD] & \\
\hline
\end{tabular}

This table shows that the female $(100 \%)$, also age $\leq 30$ years $(46.7 \%)$ and $>30$ years $(53.3 \%)$ and ranged 21-40 with mean 321.27 \pm 5.69 .

Table (2): Area liposuction distribution of the study group.

\begin{tabular}{|l|l||}
\hline Area Liposuction & Total $(\mathbf{N}=\mathbf{1 5})$ \\
\hline \hline Abdomen + arm & $1(6.7 \%)$ \\
\hline Abdomen + back & $3(20 \%)$ \\
\hline Abdomen + back + arm & $1(6.7 \%)$ \\
\hline Abdomen + back + thigh & $1(6.7 \%)$ \\
\hline Abdomen + hip & $1(6.7 \%)$ \\
\hline Abdomen + flanks + arm & $1(6.7 \%)$ \\
\hline Abdomen + thigh & $6(40 \%)$ \\
\hline Abdomen + flanks & $1(6.7 \%)$ \\
\hline
\end{tabular}

This table shows that the Abdomen + arm 1 (6.7\%), Abdomen + back $3(20 \%)$, Abdomen + back + arm $1(6.7 \%)$, Abdomen + back + thigh 1 (6.7\%), Abdomen + hip 1 (6.7\%), Abdomen + flanks $+\operatorname{arm} 1(6.7 \%)$, Abdomen + thigh $6(40 \%)$ and Abdomen + flanks $1(6.7 \%)$ of area liposuction. 
Table (3): Volume liposuction (liter) distribution of the study group.

\begin{tabular}{||l|l||}
\hline \hline Volume liposuction (Liter) & Total $(\mathbf{N}=15)$ \\
\hline \hline$\leq 7$ & $7(46.7 \%)$ \\
\hline$>7$ & $8(53.3 \%)$ \\
\hline Range [Mean \pm SD] & $5-12[7.36 \pm 1.84]$ \\
\hline
\end{tabular}

This table shows that the $\leq 7$ (46.7\%) and $>7$ (53.3\%) of volume liposuction (liter).

Table (4): Comparison between pre and after 3 months according to weight $(\mathrm{kg})$.

\begin{tabular}{|c|c|c|c|c|c|c|}
\hline \multirow[b]{2}{*}{ Weight (kg) } & \multirow[b]{2}{*}{ Range } & \multirow[b]{2}{*}{ Mean \pm SD } & \multicolumn{4}{|c|}{ Paired Sample t-test } \\
\hline & & & $\begin{array}{l}\text { Mean } \\
\text { Diff. }\end{array}$ & Change\% & t-test & p-value \\
\hline Pre & $69-92$ & $80.87 \pm 6.57$ & \multirow{2}{*}{$-3.17 \pm 1.53$} & \multirow{2}{*}{$-3.91 \pm 1.87$} & \multirow{2}{*}{3.142} & \multirow{2}{*}{$0.041^{*}$} \\
\hline After 3 months & $65-88$ & $77.70 \pm 6.36$ & & & & \\
\hline
\end{tabular}

*p-value <0.05 Significant

This table shows statistically significant low difference between pre and after 3 months according to weight $(\mathrm{kg})$.

Table (5): Comparison between pre and after 3 months according to BMI [wt/(ht)^2].

\begin{tabular}{|c|c|c|c|c|c|c|}
\hline \multirow[b]{2}{*}{ BMI [wt/(ht $\left.)^{\wedge} 2\right]$} & \multirow[b]{2}{*}{ Range } & \multirow[b]{2}{*}{ Mean \pm SD } & \multicolumn{4}{|c|}{ Paired Sample t-test } \\
\hline & & & $\begin{array}{c}\text { Mean } \\
\text { Diff. }\end{array}$ & Change \% & t-test & p-value \\
\hline Pre & $26-35$ & $30.97 \pm 2.76$ & \multirow{2}{*}{$-1.43 \pm 0.63$} & \multirow{2}{*}{$-4.66 \pm 2.19$} & \multirow{2}{*}{3.114} & \multirow{2}{*}{$0.037 *$} \\
\hline After 3 months & $25-34$ & $29.54 \pm 2.88$ & & & & \\
\hline
\end{tabular}

*p-value $<0.05$ Significant

This table shows statistically significant low difference between pre and after 3 months according to BMI [wt/(ht) $\left.)^{\wedge}\right]$.

Table (6): Comparison between pre and after 3 months according to fasting insulin.

\begin{tabular}{|c|c|c|c|c|c|c|}
\hline \multirow{2}{*}{ Insulin } & \multirow{2}{*}{ Range } & \multirow{2}{*}{ Mean \pm SD } & \multicolumn{4}{|c|}{ Paired Sample t-test } \\
\hline & & & Mean Diff. & Change \% & t-test & p-value \\
\hline Pre & $5.8-18$ & $10.21 \pm 3.49$ & \multirow{2}{*}{$-1.65 \pm 1.16$} & \multirow{2}{*}{$-16.57 \pm 9.93$} & \multirow{2}{*}{5.517799} & \multirow{2}{*}{$<0.001 * *$} \\
\hline After 3 months & $3.5-15$ & $8.56 \pm 3.08$ & & & & \\
\hline
\end{tabular}

**p-value <0.001 Highly Significant 3 months .

This table shows highly statistically significant decrease to fasting insulin between pre and after

Table (7): Volume liposuction (liter) distribution of the study group.

\begin{tabular}{||l|l||}
\hline Hb A1c & Total $(\mathbf{N}=15)$ \\
\hline \hline$<5$ & $8(53.3 \%)$ \\
\hline$\geq 5$ & $7(46.7 \%)$ \\
\hline Range [Mean \pm SD] & $4-5.8[4.79 \pm 0.59]$ \\
\hline
\end{tabular}

This table shows that the $<5(53.33 \%)$ and $\geq 5(46.7 \%)$ of $\mathrm{HbA} 1 \mathrm{c}$.

Table (8): Comparison between pre and after 3 months according to $\mathrm{Hb}$.

\begin{tabular}{||l|l|l|l|l|l|l||}
\hline \multirow{2}{*}{ Hb. } & \multirow{2}{*}{ Range } & \multirow{2}{*}{ Mean \pm SD } & \multicolumn{4}{c|}{ Paired Sample t-test } \\
\cline { 5 - 7 } & & & \multicolumn{1}{c|}{ Mean Diff. } & \multicolumn{1}{c|}{ Change\% } & p-test & palue \\
\hline \hline Pre & $10-14$ & $12.14 \pm 1.11$ & \multirow{2}{*}{$-1.91 \pm 0.72$} & $-15.89 \pm 6.30$ & 10.20592 & $<0.001 * *$ \\
\hline After 3 months & $7.8-12.5$ & $10.23 \pm 1.38$ & & & \\
\hline
\end{tabular}

**p-value $<0.001$ Highly Significant 
This table shows highly statistically significant difference between pre and after 3 months according to $\mathrm{Hb}$.

Table (9): Correlation between pre weight, insulin and $\mathrm{Hb}$, using Pearson Correlation Coefficient.

\begin{tabular}{||l|l|l|l|l||}
\hline & & Weight: After 3 months & Fasting Insulin: After 3 months & Hb: After 3 months \\
\hline \hline \multirow{2}{*}{ Weight: After 3 months } & $\mathrm{r}$ & & -0.033 & 0.014 \\
\cline { 2 - 5 } & $\mathrm{p}$-value & & 0.907 & 0.961 \\
\hline \multirow{2}{*}{ Insulin: After 3 months } & $\mathrm{r}$ & -0.033 & & 0.132 \\
\cline { 2 - 5 } & $\mathrm{p}$-value & 0.907 & & 0.640 \\
\hline \multirow{2}{*}{ Hb: After 3 months } & $\mathrm{r}$ & 0.014 & 0.132 & \\
\cline { 2 - 5 } & $\mathrm{p}$-value & 0.961 & 0.640 & \\
\hline
\end{tabular}

r-Pearson correlation Coefficient

p-value >0.05 Non Significant

This table shows no statistically significant correlation between pre weight, insulin and $\mathrm{Hb}$.

Table (10): Correlation between post weight, insulin and $\mathrm{Hb}$, using Pearson Correlation Coefficient.

\begin{tabular}{||l|l|l|l|l||}
\hline & & Weight: Pre & Insulin: Pre & Hb: Pre \\
\hline \hline \multirow{2}{*}{ Weight: Pre } & $\mathrm{r}$ & & 0.018 & -0.085 \\
\cline { 2 - 5 } & $\mathrm{p}$-value & & 0.950 & 0.763 \\
\hline \multirow{2}{*}{ Insulin: Pre } & $\mathrm{r}$ & 0.018 & & 0.196 \\
\cline { 2 - 5 } & $\mathrm{p}$-value & 0.950 & & 0.484 \\
\hline \multirow{2}{*}{ Hb: Pre } & $\mathrm{r}$ & -0.085 & 0.196 & \\
\cline { 2 - 5 } & $\mathrm{p}$-value & 0.763 & 0.484 & \\
\hline
\end{tabular}

$r$-Pearson correlation Coefficient

p-value >0.05 Non Significant

\section{Discussion}

This table shows no statistically significant correlation between post weight, insulin and $\mathrm{Hb}$.

There are multiple researches investigated the effects of liposuction on metabolism.

According to meta-analysis done in 2004, a general improvement in fasting plasma insulin appears to be influenced by large volume liposuction in obese women. Importantly, high levels of fasting plasma insulin are significantly correlated with cardiovascular mortality ${ }^{\mathbf{( 1 1 )}}$.

Giese et al. ${ }^{(12)}$ by performing the same type of surgery, noted a significant decrease in weight, systolic blood pressure, and fasting insulin levels 4 months after liposuction. These general health improvements were still maintained at the 1 year follow-up assessment.

Much research on insulin sensitivity after liposuction has been performed during the last decade. The first study on this specific topic was performed in 2004 by Giugliano et al. ${ }^{(13)}$ via measurement of the Homeostasis Model Assessment $(\mathrm{HOMA}=$ fasting plasma glucose 9 fasting serum insulin divided by 25) as well as serum adiponectin and circulating inflammatory markers (interleukin-6 [IL-6], IL-18, tumor necrosis factor-alpha [TNFa], and C-reactive protein [CRP]) in obese and normal weight women treated with liposuction. These authors demonstrated that in obese women, liposuction is associated with amelioration of insulin resistance and reduces circulating markers of vascular inflammation and decrease fasting insulin levels.

The 1-month insulin sensitivity results are similar to several previous studies that have shown that large-volume liposuction improves insulin sensitivity as measured using either fasting parameters or an insulin tolerance test ${ }^{(14)}$.

Insulin sensitivity also has been analyzed through HOMA by other authors such as Busetto et al ${ }^{(15)}$ and Ybarra et al. ${ }^{(16)}$ In 2008, Bassetto et al. studied 15 premenopausal obese women treated with surgical removal of subcutaneous fat (ultrasound-assisted megalipoplasty [UAM]). This procedure was proved to be associated with an acute inflammatory reaction with a high resting 
metabolic rate (REE) and insulin resistance. However, the metabolic effects of fat mass removal appeared later with a reduction of leptin levels and REE and an improvement in insulin resistance, decrease fasting insulin levels.

Also in 2008, Ybarra et al. (16) recruited 20 healthy volunteers ( 2 men and 18 women) $39.6 \pm 7.7$ years of age with a BMI of $25.3 \pm 4.7$ who were treated with abdominal liposuction. At 4 months after surgery the patients had improved major lipoprotein components of obesity-associated dyslipidemia (free fatty acids, very-lowdensity lipoprotein [VLDL], HDL, triglycerides) but showed no change in insulin sensitivity (HOMA, glucose, or insulin concentrations).

On the contrary, in 2005, D'Andrea et al. (14) evaluated insulin sensitivity by measuring another parameter: the hyperinsulinemic glucose clamp. These authors studied 123 obese women treated with liposuction and found that 3 months after surgery, insulin sensitivity improved, as well as inflammatory markers and lipid levels, decrease fasting insulin levels.

Davis et al. (17) analyzed 15 overweight or obese women and showed that 1 month after surgery, insulin sensitivity (glucose tolerance test) improved, decrease fasting insulin levels in obese patients but not in overweight patients.

Hernandez et al. ${ }^{(18)}$ analyzed 32 women with a BMI higher than $22 \mathrm{~kg} / \mathrm{m} 2$ (14 treated with liposuction and 18 serving as control patients without surgery). By analyzing lipid assessment and hyperinsulinemiceuglycemic clamp, these authors showed no metabolic amelioration even 6 months after surgery. They also noted that 1 year after thigh liposuction, fat tissue was restored and redistributed from the thigh to the abdomen.

In 2004 Klein et al. ${ }^{(19)}$ followed 15 obese women treated with liposuction. They found that surgery did not significantly improve the cardiovascular risk factors (blood pressure, lipid levels, plasma glucose, plasma fasting insulin) or indicators of inflammation.

In study done in 2002 measuring fasting insulin levels, lipid and lipoproteins levels, indices associated with glucose tolerance and blood pressure, in 34 obese patients treated with liposuction and abdominoplasty. These values were measured preoperatively, then 3 and 12 months postoperatively, after which they were compared with equivalent data for 23 control patients treated with breast reduction. This study showed that lipectomy reduced plasma insulin levels but had no lasting effect on plasma lipids (10).

In general, all the studies showed that some indicators, such as lipid levels and insulin sensitivity, fasting insulin levels, have a more stable trend. In fact, whereas lipid levels seemed to show no improvement with surgery, insulin sensitivity and fasting insulin levels, inflammatory markers seemed to be improved after large-volume liposuction. The consistently reported perioperative variation of insulin levels and sensitivity appears to support the theory that adipose tissue removal improves insulin balance. In obese healthy women, fasting plasma insulin may be reduced by large-volume liposuction. The pathogenesis of insulin resistance in metabolic syndrome is associated with the expansion of the adipose tissue mass. In metabolic syndrome, adipocytes either are implicated with excessive flux of fatty acids or contribute to the creation of a proinflammatory state by producing proinflammatory cytokines, both of which are conditions that determine insulin resistance ${ }^{(\mathbf{2 0})}$.

In the majority of the studies that explored the effects of liposuction and dermolipectomy on metabolism, insulin sensitivity appeared to increase and inflammatory markers, fasting insulin levels to decrease after surgery. This aspect can be seen as a backwards way of metabolic syndrome pathogenesis. In metabolic syndrome, the expansion of adipose tissue is one cause of insulin resistance, whereas the adipose tissue decreases after liposuction and may determine the increase of insulin sensitivity. Peripheral adipose tissue is known to be involved to a lesser degree than visceral fat in the body's metabolic balance. However, the total body adipose mass and its relevant variation may have measurable effects on metabolic variables and indicators.

The question whether liposuction has a positive metabolic effect or not is still open and deserves more research. Moreover, the medical literature does not mention any comparison between patients treated with dermolipectomy and patients treated with liposuction with regard to any difference in metabolic variations caused by either procedure.. Therefore, such a comparison should be encouraged as a future investigation. 
Benatti et al. (21) noted that we still cannot give a final answer to these issues, but exercise training associated with liposuction or dermolipectomy may improve insulin sensitivity and inflammatory balance.

On the other hand, lipectomy might sometimes be followed by a regain of fat because fat mass is physiologically regulated. Hernandez et al. (18) noted an abdominal redistribution of fat using long-term Dexa scans on patients who had been treated with liposuction. It is possible that regaining fat over time after liposuction may induce insulin resistance due to a reduction in the number of adipocytes removed with lipoaspiration. Due to the limited size of the adipocyte increase, a reduction in the number of fat cells might favor a deposition of ectopic fat and some degree of insulin resistance.

It is essential to underscore that liposuction must not be seen as a shortcut to solve obesity and related diseases but that it can be a useful aid in the multidisciplinary treatment of these diseases. Most importantly, the surgical removal of fat is not detrimental with regard to variation of metabolic indices. Prospective studies, possibly including randomized controlled trials and laboratory research, are necessary to assess changes in metabolic parameters (glycemia, insulin levels) in a homogeneous patient population undergoing liposuction or dermolipectomy for a better comprehension of the relationship between plastic surgery and cardiovascular or metabolic status ${ }^{(22)}$.

\section{Conclusion}

- The analysis of the study suggests that plastic surgery could play a role in metabolism.

- It is essential to underscore that liposuction must not be seen as a shortcut to solve obesity and related diseases but that it can be a useful aid in the multidisciplinary treatment of metabolic diseases. Most importantly, the surgical removal of fat is not detrimental with regard to variation of metabolic indices

- Prospective studies, possibly including randomized controlled trials and laboratory research, are necessary to assess changes in metabolic parameters (glycemia, insulin levels) in a homogeneous patient population undergoing liposuction for a better comprehension of the relationship between plastic surgery and cardiovascular or metabolic status.

\section{References}

1. Coleman WP, 3rd, Glogau RG, Klein JA, Moy RL, Narins RS, Chuang TY et al. (2001): Guidelines of care for liposuction. J Am Acad Dermatol., 45:438-47.

2. Matarasso $A$ and Hutchinson $\mathbf{O H}$ (2001): Liposuction. JAMA., 285:266268.

3. Cárdenas-Camarena L, Gerardo LP, Durán H, Bayter-Marin JE (2017): Strategies for Reducing Fatal Complications in Liposuction. Plastic and Reconstructive Surgery Global Open, 5(10).

4. Narsete $T$, Narsete $M$, Buckspan $R$, Ersek R (2012): Large-volume liposuction and prevention of type 2 diabetes: a preliminary report. Aesthetic plastic surgery, 36(2):438-42.

5. Stephan PJ and Kenkel JM (2010): Updates and advances in liposuction. Aesthet. Surg. J., 30:83-97.

6. Iverson RE, Lynch DJ (1996): American society of plastic surgeons committee on patient safety. Plast Reconstr Surg., 113(5):1478-90.

7. Hunstad JP(1995) Body contouring in the obese patient resulting in pulmonary fat embolism syndrome. Clin Plast Surg. , 23:647-70.

8. Johnson J, Duick $D$, Chui $M$ and Aldasouqi $S$ (2010): identifying prediabetes using fasting insulin levels. Endocrine Practice, 16(1): 47-52.

9. Harris MI, Cowie CC, Gu K, Francis $M E$, Flegal $K$ and Eberhardt $M S$ (2002): Higher fasting insulin but lower fasting C-peptide levels in African Americans in the US population. Diabetes Metab Res Rev., 18(2):149-55.

10. Gonza'lez-Ortiz M, Robles-Cervantes JA, Ca'rdenas-Camarena L, BustosSaldaña R, Martı'nez-Abundis E (2002): The effects of surgically removing subcutaneous fat on the metabolic profile and insulin sensitivity in obese women after large-volume liposuction treatment. Horm Metab Res., 34:446-449.

11. Hu G, Qiao Q, Tuomilehto J, Eliasson $M$ et al. (2004): Plasma insulin and cardiovascular mortality in nondiabetic European men and women: a meta- 
analysis of data from eleven prospective studies. Diabetologia, 47:1245-1256.

12. Giese SY, Bulan EJ, Commons GW, Spear SL, Yanovski JA (2001): Improvements in cardiovascular risk profile with large volume liposuction: a pilot study. Plast Reconstr Surg 108: 510-519.

13. Giugliano G, Nicoletti G, Grella E, Giugliano F, Esposito K, Scuderi N et al. (2004): Effect of liposuction on insulin resistance and vascular inflammatory markers in obese women. Br J Plastic Surg., 57: 190-194.

14. D'Andrea F, Grella R, Rizzo MR, Grella E, Grella R, Nicoletti G, Barbieri M, Paolisso G (2005): Changing the metabolic profile by large-volume liposuction: a clinical study conducted with 123 obese women. Aesthetic Plast Surg., 29:472-478.

15. Busetto L, Bassetto F, Zocchi M, Zuliani F, Nolli ML, Pigozzo S, Coin A, Mazza M, Sergi G, Mazzoleni F, Enzi G (2008): Effects of the surgical removal of subcutaneous adipose tissue on energy expenditure and adipocytokine concentrations in obese women. Nutr Metab Cardiovasc Dis., 18:112-120.

16. Ybarra J, Blanco-Vaca F, Ferna'ndez $S$, Castellv' A, Bonet R, Palomer X, Ordo'n ez-Llanos J, Trius A, VilaRovira R, Pe'rez A (2008): The effects of liposuction removal of subcutaneous abdominal fat on lipid metabolism are independent of insulin sensitivity in normal-overweight individuals. Obes Surg.,18:408-414.

17. Davis DA, Pellowski DM, Davis DA, Donahoo WT (2006): Acute and 1month effect of small-volume suction lipectomy on insulin sensitivity and cardiovascular risk. Int $\mathrm{J}$ Obes London, 30: 1217-1222.

18. Hernandez, Kittelson J, Law $\mathrm{C}$ et al.(2011): Defense of Body Fat and Patterns of Restoration. Obesity, 19: 1388-1395.

19. Klein S, Fontana L, Young VL, Coggan AR, Kilo C, Patterson BW, Mohammed BS (2004): Absence of an effect of liposuction on insulin action and risk factors for coronary heart disease. $\mathrm{N}$ Engl J Med., 350:2549-2557.

20. Eckel RH, Grundy SM, Zimmet PZ (2005): The metabolic syndrome. Lancet, 365:1415-1428.

21. Benatti FB, Lira FS, Oyama LM, do Nascimento CM, Jr Lancha AH (2011): Strategies for reducing body fat mass: effects of liposuction and exercise on cardiovascular risk factors and adiposity. Diabetes Metab Syndr Obes., 4:141-154.

22. Dandona P, Aljada A, Bandyopadhyay A (2004): Inflammation: the link between insulin resistance, obesity, and diabetes. Trends Immunol., 25:4-7. 